\title{
Features of the Influence of the Technology of Vibration Simulators on the Physical and Functional Characteristics of the Female Handball Players of Adolescence and Early Youth Age in the Recovery Period
}

\author{
Viktor Gorelik ${ }^{1, a *}$, Svetlana Filippova², Yuri Matveev², Valeria Malysheva ${ }^{3}$
}

1 Department of Adaptive Physical Education, Sport and Tourism, Institute of Physical Culture and Sports, Togliatti State University, Togliatti, Russia

\begin{abstract}
2 Department of Adaptive Physical Culture and Biomedical Disciplines, Institute of Natural Sciences and Sports Technologies, Moscow City Pedagogical University, Moscow, Russia
\end{abstract}

3 The Studio of Personal Trainings and Rehabilitation, Togliatti, Russia

\author{
alecgoy@list.ru \\ ${ }^{*}$ Corresponding author
}

\begin{abstract}
Keywords: physical education, pedagogical approaches in training, youth sport, handball, recovery period, flexibility, coordination abilities, heart rhythm, vibration simulator, motor analyzer, motor actions, amplitude of movements, muscle tension, stretching, relaxation
\end{abstract}

\begin{abstract}
The paper discusses results of studies focused on the impact of training on vibration stimulators on the recovery process in post-training period in female handball players of 13-14 and 15-16 years of age. Dynamics of physical qualities (flexibility, coordination of movements) and functional indices of the cardiovascular system (CVS) have been determined. The developing and training influence of vibration training technology on physical qualities and functional indices is demonstrated, which is based on the activation of the processes of restoration of the body's regulatory systems, its reserve capabilities, and the overall development of the organism that continues in adolescence. The obtained data allow to consider the effective technology of vibration machines for post-training recovery of young athletes and serve as a justification for the wider use of vibration simulators in the training process of handball players.
\end{abstract}

\section{Introduction}

The training process in contemporary handball from the initial stage of preparation to the training of a highlevel athlete represents a well-developed system of interrelated stages that contributes to the achievement of planned sports results $[2,3,4]$. At all stages, including the initial training (IT), the training stage (TS), improving sports skills (ISS), as well as the level of higher sportsmanship (HS), it is necessary to take into account certain age features, the functional state of the athlete's organism, depending on the integrative activity of neurophysiological and psychophysiological regulatory systems, the level of development of motor actions in solving game problems in the variational conditions of gaming activity $[4,5,6,8]$.

The choice of this topic is due to the fact that the issues of training young athletes, handball players with the use of contemporary and advanced health-improving fitness approaches are not sufficiently considered and well-studied, both theoretically and methodologically, including in the practice of training [2, $3,4]$.

Features of the physiology of the central nervous system (CNS), which manages the locomotion of athletes in gaming, requires optimization of the functions of sensory systems, the stability of proprioceptive afferentation, developed coordination abilities, and the balance of muscular efforts in the implementation of gaming motor actions $[4,14]$. These factors, taken all together, lead to an increase in the regulatory, motor, functional, and reserve capabilities of young handballers, contribute to the implementation of variable power movements, the economic alternation of active and passive phases in gaming activities $[14,15,16]$.

The urgency of the research is grounded in the fact that the training process requires a high level of psychophysical readiness from sportswomen of adolescent and early youth with significant physical exertion. Therefore, achieving a sporting result is impossible without an effective regenerating of the 
developing organism in the post-training period $[4,5,6]$.

The use of medico-biological technology of vibration simulators during the recovery period after significant physical exertion of athletes has shown its promise in various sports [9]. However, the peculiarities of using this technology in handball, which has a number of psychophysical specifics in the area of training loads and the recovery process, have not been studied enough. Consequently, research in this area is relevant, especially given the significant role of the recovery period for maintaining health, preventing maladaptive border states, and thereby reducing the risks of injury in youth female handball as a sport discipline $[5,6,14]$.

\section{Methods and organization of the study}

The study was conducted in the form of a pedagogical experiment, which covered 80 female handball players being divided into 2 groups taking into account their age: the average age of 13-14 years (group 1) and the senior age of 15-16 years (group 2).

The main stages of the study lasted from November 2017 to June 2018.

At the first, preparatory, stage (November 2017 - December 2017), groups and methods of research were selected.

At the second stage (January 2018 - June 2018), experimental pedagogical studies were conducted, including the ascertaining, forming, and control stages.

\section{The ascertaining stage.}

At the ascertqining stage of the experiment, all subjects were divided into two groups: the main group (MG) of 20 people for the middle and older age (two groups in total). They performed specially selected exercises to relieve muscle tension, develop flexibility and coordination abilities on the Power Plate in the Studio of Personal Training and Rehabilitation of Power Plate. Comparison groups (CG) included 20 people for each age group 1 and 2, who had a standard training program twice a week in the Olympus sports complex. Comparison of the $\mathrm{MG}$ and $\mathrm{CG}$ results made it possible to obtain objective data on the investigated problem.

The training process in the four groups was held for 6 months, according to the program of the IT stage for the $1^{\text {st }}$ group, and the TS program was used for the 2 nd group. Then, at the control stage of the experiment, the flexibility and coordination abilities of the MG and CG handball players were compared again.

2. The forming stage. During the experiment, MG used a set of exercises on a special vibration platform to relieve muscle tension, develop flexibility and coordination abilities of young handball players. In the $\mathrm{CG}$, girls were engaged in stretching during additional training in the post-training period without the use of a vibration platform.

3. The control stage. At the final stage of the pedagogical experiment, the final testing of the athletes was conducted. At the third stage (June 2018 - July 2018), we focused on data processing, interpretation of the research results, formulation of conclusions. For data processing, the Statistica program was used, in which the parametric t-Student criterion for independent and dependent samples was determined.

\section{Technique of using the Power Plate vibration simulator}

The study used the technology of vibration stimulator, in which the platform of the Power Plate simulator creates three-dimensional fluctuations in the physiological frequency, induces involuntary muscle contractions up to 40 times per second, and up to $100 \%$ of the muscle fibers are activated.

The technique of training on the Power Plate simulators has a number of advantages: accelerating blood circulation, increasing muscle strength and increasing flexibility, expanding the range of motion, increasing bone density, reducing pain, and facilitating the rapid recovery of the body. This technology is widely used in medicine to prevent and treat various types of diseases and injuries. The simulator is suitable for people of all age categories, life styles, and physical abilities [9]. 


\section{A technique for diagnosing the functional state of an organism using the hardware-software complex "Varicard 2.51"}

The hardware and software complex "Varikard 2.51" is designed for processing cardiointervalograms for the purpose of analyzing the heart rate variability and assessing the state of the autonomic nervous system (ANS).

Based on the data of vegetative balance and neurohumoral regulation obtained with the help of the "Varicard 2.51" complex, the functional state of the organism of handballers was evaluated. The standard analysis protocol is carried out on 5-minute recording areas [10].

\section{Method for determining the muscle tone by a sports doctor}

The muscle tone can be defined palpably. During a deep palpation in consistency, healthy muscles should be soft, painless, and elastic. Because of physical and psychoemotional stress, pathological conditions, the consistency of muscles changes. There are three degrees of increase in muscle tone $(\mathrm{T})$ :

(1) I degree: a slight increase in $\mathrm{T}$; with palpation, the muscle is soft, fingers are relatively easy to sink into it;

(2) II degree: a marked increase in $\mathrm{T}$; with palpation, the muscle is of moderate density, but the fingertips can be immersed in its thickness with a certain effort;

(3) III degree: a pronounced increase in T; the muscle is of "rocky" density, it is impossible or almost impossible to deform it by palpation.

With the help of examination and palpation, violations of trophic and muscle tone are established, fibrillations and fasciculations are detected according to I. P. Antonov and Ya. A. Lupian [1].

In order to study the increase in mobility of joints (flexibility) by means of stretching (sets of exercises for stretching the musculoskeletal system (MS), the following motor tests were used at the ascertaining and control stages of the experiment.

\section{Motor tests:}

1. "Test with a gymnastic stick": the test is aimed at assessing the mobility of the shoulder joints (in cm).

The subject, holding the ends of the gymnastic stick, performs the straightening of the hands straight back. Mobility in the shoulder joint is assessed by the distance between the hands at the twisting time: the smaller the distance, the higher the mobility in this joint.

\section{2. "Tilting forward": the test is aimed at measuring the flexibility of the spine (in cm).}

Tilting forward from a standing position with straight legs is performed from the following starting position: standing on a gymnastic bench, the legs are straightened in the knees, the feet are placed parallel in a width of $10-15 \mathrm{~cm}$. When performing the test on the floor, the subject performs two preliminary tilts on command. At the third slope, she freezes at the bottom point, and the result is fixed for 2 seconds.

\section{3. "Bridge": the test is aimed at measuring the flexibility of the spine (in $\mathrm{cm}$ ).}

The athlete performs the exercise called the "bridge" with the support of hands and feet. The result (in $\mathrm{cm}$ ) is measured from the heels to the fingertips of the subject. The smaller the distance, the higher the level of flexibility, and vice versa.

\section{4. "Cross-twine": the test is aimed at measuring mobility in the hip joint (cm).}

The subject performs the exercise "cross-twine" with the maximum dilution of the legs to the sides, with the support of the hands. The level of mobility in this joint is estimated from the distance from the floor to the pelvis (coccyx): the smaller the distance, the higher the level of flexibility, and vice versa.

To determine the coordination abilities, the following locomotor motor tests were used in the ascertaining and control stages of the experiment. 


\section{Locomotive motor tests:}

\section{Running 10 meters with the ball only with the right or left hand (s)}

Running a stretch of 10 meters with leading the ball with the leading hand for a while.

\section{Throwing a tennis ball at a distance from the position of "sitting-feet-apart" with a leading} hand (m)

From the position called "sitting-feet-apart", the athlete throws a tennis ball at a range.

3. Running with the maximum frequency of steps in place and moving for 15 seconds (the number of steps)

The athlete performs the maximum number of steps in place and moving over a certain time.

4. Jerking on the visual signal: after the partner, in competition with the partner for the flying ball, and catching the ball (out of 30 seconds)

The athlete performs a movement for a partner for the flying of the ball on time.

\section{Pedagogical experiment}

The pedagogical experiment lasted for 6 months. The training was held 2 times a week and consisted of 3 parts listed below (Table 1): (1) the introductory part (warm-up, 30Hz / 30s) for 2-3.5 min; the main part (in which exercises are used to stretch the muscles of the whole body, $30 \mathrm{~Hz} / 30 \mathrm{~s}$ ) for 18-20 min; (3) the final part (massage, $40 \mathrm{~Hz} / 60 \mathrm{~s}$ ) for $4 \mathrm{~min}$.

Table 1 shows the exercises performed by female handball players at the forming stage of the pedagogical experiment: MG1 - "adolescence"; MG2 - "early youth."

TABLE 1. EXERCISES WITH A VIBRATION STIMULATOR USED IN THE MG.

\begin{tabular}{|c|c|}
\hline Name of exercise & Area of influence \\
\hline \multicolumn{2}{|c|}{ (1) Introductory part (warm-up, 30Hz/30s) for 2-3,5 minutes } \\
\hline Half-sided & Legs, buttocks, back \\
\hline Tilting forward & Back thigh \\
\hline Lungs & $\begin{array}{l}\text { Anterior thigh } \\
\text { (on each side) }\end{array}$ \\
\hline "Butterfly" & Inner thigh \\
\hline \multicolumn{2}{|c|}{ (2) The main part $(30 \mathrm{~Hz} / 30 \mathrm{~s})$ for $18-20$ minutes } \\
\hline Push-up & Thoracic muscles, shoulders, triceps \\
\hline $\begin{array}{c}\text { Sitting in Turkish } \\
\text { (with legs bent and crossed in front, back } \\
\text { straight) }\end{array}$ & Wide muscle back \\
\hline Tilting to the sitting leg & $\begin{array}{c}\text { Hamstring } \\
\text { (per foot) }\end{array}$ \\
\hline Balance & $\begin{array}{l}\text { Press, back } \\
\text { (on each side) }\end{array}$ \\
\hline Stretching calves & Calf muscles \\
\hline "Dog" & $\begin{array}{c}\text { Inner thigh } \\
\text { (per foot) }\end{array}$ \\
\hline Stretching buttocks & $\begin{array}{l}\text { Buttocks } \\
\text { (per foot) }\end{array}$ \\
\hline Moving legs in sides & Inner thigh \\
\hline Lateral slope & $\begin{array}{l}\text { Side surface } \\
\text { (on each side) }\end{array}$ \\
\hline Hands back & Shoulder \\
\hline "Cat" & Thoracic area \\
\hline Scapula & Scapula \\
\hline Pulling the neck & Neck, back \\
\hline Pulling the body & Shoulders, back \\
\hline \multicolumn{2}{|c|}{ (3) The final part (massage, $40 \mathrm{~Hz} / 60 \mathrm{~s}$ ) for 4 minutes } \\
\hline
\end{tabular}




\begin{tabular}{|c|c|}
\hline Thigh back p massage & Hind femora \\
\hline Front thigh massage & Anterior thigh \\
\hline Relaxation of the back & Back \\
\hline Massage of gastrocnemius muscles & Calf muscles \\
\hline
\end{tabular}

\section{Results}

The study is devoted to the use of training on a vibration simulator with the elements of stretching and subsequent relaxation of teenagers in the MG 1 and 2. Such lessons give not only health (restoring the body's reserves) in the post-training period, but also develop and train strong characteristics in athletes [4, 5, $6,9]$. Perfected psycho-physical qualities and abilities of athletes: muscle and psychoemotional tension decreases, joint mobility (flexibility), coordination abilities, and strength qualities increase. This ensures maximum amplitude of movements, freedom of movement and ballistic maneuver, more effective low protective rack. The development of motor abilities in handball players contributes to improving their technical preparedness, tactical improvement of team interactions $[2,3,4,5]$.

In the adolescent period, the improvement of motor abilities and motor qualities continues. At this age, there are signs of stress in girls, as well as an unstable psychoemotional condition, which can improve under the influence of systematic sports activities. For this period, the oscillatory character of the regulatory influence of the VNS (inhibition and excitation processes) is characteristic. As a result, the motor skill can be developed with errors, technical aspects of exercises are not accurately performed. To prevent dysregulations, it is necessary to dose the load and rest intervals $[6,11,12,14,15]$.

In adolescence, active growth of the body continues, body size increases, and when an early young adult begins growing, the growth processes slow down. During this period, power and motor qualities and coordination abilities are improved $[6,11,12]$. In adolescence, the lower functional capabilities of the CVS and the respiratory system are observed, which is caused by the incomplete formation of anatomic and morphological structures and regulatory mechanisms of the cardiorespiratory system. Therefore, in adolescence and early youth, it is impossible to force the load. Moreover, this can lead to a disruption in the psychophysical adaptation of a young athlete in the training process $[6,11,12,15,16]$.

The Table 2 presents results of diagnostics of the physical quality "flexibility" in the "adolecence" (1) and "early youth" (2) MGs and CGs of handball players.

TABLE 2. ASSESSING THE FLEXIBILITY IN CARRYING OUT THE PEDAGOGICAL EXPERIMENT IN THE OG AND GS FOR THE 1ST AND 2ND GROUPS: “ADOLESCENCE” (GROUP 1) AND “EARLY YOUTH” (GROUP 2).

\begin{tabular}{|c|c|c|c|c|c|c|c|c|}
\hline & \multicolumn{3}{|c|}{ MG } & \multicolumn{3}{c|}{ CG } \\
\hline $\begin{array}{c}\text { Research } \\
\text { stage }\end{array}$ & $\begin{array}{c}\text { Test with a } \\
\text { gymnastic } \\
\text { stick (cm) }\end{array}$ & $\begin{array}{c}\text { Tilting } \\
\text { forward } \\
(\mathrm{cm})\end{array}$ & $\begin{array}{c}\text { Cross-twine } \\
(\mathrm{cm})\end{array}$ & $\begin{array}{c}\text { Bridge } \\
(\mathrm{cm})\end{array}$ & $\begin{array}{c}\text { Test with a } \\
\text { gymnastic } \\
\text { stick (cm) }\end{array}$ & $\begin{array}{c}\text { Tilting } \\
\text { forward } \\
(\mathrm{cm})\end{array}$ & $\begin{array}{c}\text { Cross-twine } \\
(\mathrm{cm})\end{array}$ & $\begin{array}{c}\text { Bridge } \\
(\mathrm{cm})\end{array}$ \\
\hline $\begin{array}{c}\text { Ascertaining } \\
\text { stage } \\
\text { (1st group) }\end{array}$ & $61,5 \pm 0,6$ & $25,8 \pm 0,8$ & $45,7 \pm 1,1$ & $50,6 \pm 0,4$ & $60,6 \pm 0,6$ & $26,4 \pm 0,6$ & $46,8 \pm 1,3$ & $52,4 \pm 0,7$ \\
\hline $\begin{array}{c}\text { Ascertaining step } \\
\text { (2nd group) }\end{array}$ & $59,3 \pm 0,3$ & $27,1 \pm 0,4$ & $44,9 \pm 0,4$ & $52,6 \pm 0,5$ & $59,6 \pm 0,6$ & $26,5 \pm 0,8$ & $\begin{array}{c}46,6 \pm 0,4 \\
51,3 \pm 0,9\end{array}$ \\
\hline $\begin{array}{c}\text { Control stage } \\
\text { (1st group) }\end{array}$ & $54,7 \pm 0,5$ & $19,7 \pm 0,5$ & $39,6 \pm 0,5$ & $45,2 \pm 0,4$ & $56,2 \pm 0,4 *$ & $\begin{array}{c}23,4 \pm 0,6 \\
* *\end{array}$ & $\begin{array}{c}43,2 \pm 0,4 \\
* *\end{array}$ & $\begin{array}{c}48,3 \pm 0,3 \\
* *\end{array}$ \\
\hline $\begin{array}{c}\text { Control stage } \\
\text { (2ndgroup) }\end{array}$ & $55,6 \pm 0,4$ & $22,2 \pm 0,6$ & $40,1 \pm 0,7$ & $46,4 \pm 0,4$ & $57,3 \pm 0,5$ & $23,7 \pm 0,7 *$ & $\begin{array}{c}43,9 \pm 0,5 \\
* *\end{array}$ & $\begin{array}{c}49,2 \pm 0,6 \\
* *\end{array}$ \\
\hline
\end{tabular}

Significance level: * $\mathrm{p} \leq 0,05, * * \mathrm{p}<0,01$

A comparative analysis of the data in Table 2 shows that there are no significant differences between the MGs and CGs at the ascertaining stage, and the age factor also does not significantly affect the athletes' flexibility indicators when comparing the average values of groups 1 and 2 . Whereas the use of exercises on the vibrating drill during the post-training period at the forming stage in the MG led to a significant improvement in "flexibility," which 
was more pronounced in athletes of younger age (MG 1) in comparison with handballers of 15-16 years (MG 2), making the total of $5.8 \mathrm{~cm}$ and $3.9 \mathrm{~cm}$, respectively. Reliable dynamics speaking in favor of making progress in athletes' "flexibility" in athletes of the CG $(1,2)$ were not detected. These data testify to the developmental impact of the exercises on the vibration simulator on the background of its restoring influence in the post-training period on the organism.

The Table 3 shows the results of diagnosis in the conduct of coordination abilities in the "adolescent" (1) and "early youth" (2) MGs and CGs.

TABLE 3. INDICATORS OF COORDINATION ABILITIES OF THE PEDAGOGICAL EXPERIMENT IN THE MG AND CG FOR THE GROUPS 1 AND 2 OF HANDBALLERS IN “ADOLESCENCE” AND “EARLY YOUTH” AGE.

\begin{tabular}{|c|c|c|c|c|c|c|c|c|}
\hline & \multicolumn{4}{|c|}{ MG } & \multicolumn{4}{c|}{ CG } \\
\hline Research Phase & 1 & 2 & 3 & 4 & 1 & 2 & 3 & 4 \\
\hline $\begin{array}{c}\text { Ascertaining stage } \\
\text { (1 st group) }\end{array}$ & $7,3 \pm 0,08$ & $4 \pm 0,09$ & $50 \pm 0,4$ & $26 \pm 0,2$ & $7 \pm 0,04$ & $4,2 \pm 0,08$ & $51 \pm 0,08$ & $27 \pm 0,3$ \\
\hline $\begin{array}{c}\text { Ascertaining stage } \\
\text { (2nd group) }\end{array}$ & $6,6 \pm 0,5$ & $4,5 \pm 0,8$ & $54 \pm 0,9$ & $23 \pm 0,7$ & $6,5 \pm 0,9$ & $4,3 \pm 0,7$ & $55 \pm 0,5$ & $23 \pm 0,8$ \\
\hline Control stage (1st group) & $6,6 \pm 0,06$ & $5,5 \pm 0,14$ & $61 \pm 1,1$ & $21 \pm 0,3$ & $\begin{array}{c}6,9 \pm 0,09 \\
* *\end{array}$ & $5 \pm 0,18 *$ & $59 \pm 1,2$ & $\begin{array}{c}26 \pm 0,2 \\
* *\end{array}$ \\
\hline Control stage (2nd group) & $6,2 \pm 0,04$ & $6,7 \pm 0,2$ & $65 \pm 0,4$ & $18 \pm 0,7$ & $6,4 \pm 0,03 *$ & $\begin{array}{c}6,1 \pm 0,1 \\
* *\end{array}$ & $63 \pm 0,3 *$ & $21 \pm 0,9 *$ \\
\hline
\end{tabular}

1 - Running 10 meters with the ball only with the right or left hand (s).

2 - Throwing a tennis ball at a distance from the position of "sitting-feet-apart" with a leading hand (m).

3 - Running with the maximum frequency of steps in place and moving for 15 seconds (the number of steps).

4 - Jerking on the visual signal: after the partner, in competition with the partner for the flying ball, and catching the ball (out of 30 seconds).

Comparison of the data on athletes of the same age in the MG and CG in the ascertaining stage of the pedagogical experiment did not reveal any significant differences between the characteristics of their coordination abilities. At the forming stage, the MG had to a significant improvement in the "coordination abilities." Inter-age differences indicate a better formation of coordination abilities in older athletes.

The young female athletes have the 1st degree of muscle tone. Classes taught according to the standard program in the $\mathrm{CG}$ are less effective than it is on vibration simulators in the $\mathrm{MG}$ for the development of coordination abilities. This is possibly due to a more pronounced restorative effect of vibration in the post-training period.

To assess the status of regulatory systems of handball players, the variability of the heart rate (HRV) of the hardware and software complex "Varicard" was used in the study of the MG and CG, who were engaged in various programs during the post-training period [10]. The data obtained are presented in the Table 4 and Figure 1.

TABLE 4. TESTING HRV INDICATORS AT THE DETECTING STAGE OF PE IN THE MG AND CG.

Main indicators of heart rate rhythm

\begin{tabular}{|l|l|l|}
\hline & Value & Norm \\
\hline HR, bits/sec & $97^{* *}$ & $55-80$ \\
\hline SDNN, ms & 56.1 & $30-100$ \\
\hline CV (coefficient of variation), \% & 9.1 & $3-12$ \\
\hline SI (Stress Index), points & $298^{* *}$ & $50-150$ \\
\hline IC (Index of centralization) & $0.8^{* *}$ & $2-8$ \\
\hline NTI, points (2, -1) & 3 & $1-3$ \\
\hline NArr, \% & 0.0 & $0-4$ \\
\hline Strength HF, \% & $55.1^{* * *}$ & $10-30$ \\
\hline Strength LF, \% & 24.8 & $15-45$ \\
\hline Strength VLF, \% & 20.1 & $20-60$ \\
\hline TR, ms2 & 1140 & $800-1500$ \\
\hline
\end{tabular}

Atypical index (AI)

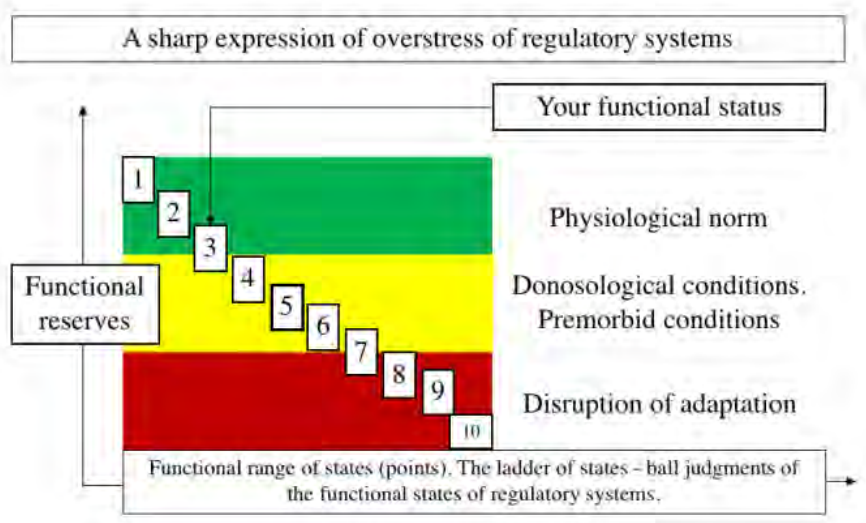


Assessment of the state of regulatory systems

\begin{tabular}{|c|c|}
\hline \multicolumn{2}{|l|}{ Level of functioning } \\
\hline Normocardia & 0 \\
\hline \multicolumn{2}{|l|}{ Stability of regulation } \\
\hline Increased stability of the rhythm & 1 \\
\hline \multicolumn{2}{|l|}{ Vegetative homeostasis } \\
\hline Increased predominance of the sympathetic nervous system & 1 \\
\hline \multicolumn{2}{|l|}{ Activity of the sympathetic vascular center } \\
\hline Moderately reduced activity of the vascular center & -1 \\
\hline \multicolumn{2}{|l|}{ Degree of centralization } \\
\hline Normal activity of central levels of regulation & 0 \\
\hline
\end{tabular}

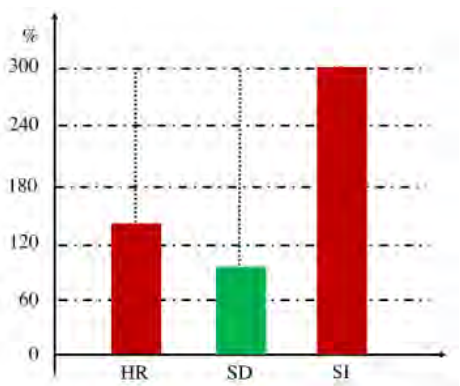

Fig. 1. Assessing the state of regulatory systems in the MG and GC at the ascertaining stage.

The functional state in the groups under the study at the asserting stage of the pedagogical experiment in both MG and CG refers to the physiological norm (3 is the functional state class of regulatory systems). However, despite the favorable evaluation of regulation systems for handball players, it is important to pay attention to the increased values of heart rate ( 97 beats per minute), as compared to the upper limit of the norm. There is also an increase of more than 2 times (in comparison with the normative level of the value) in the stress index Si. (The stress index is 298 conventional units, the norm is 50-150 conventional units). These deviations of the main characteristics of the CVS condition of the athletes testify to the intense activity of the CVS under the influence of sports training. Training loadings are performed by girls with an additional voltage of the neuroregulatory systems of the SNS and VNS. In this case, athletes should make greater efforts and expenses of psychophysical reserve capabilities for providing gaming activities.

Data on the state of regulatory systems in young handballers, obtained in the MG at the control stage after training on the vibration simulator, are presented in Table 5 and in Figure 2.

\section{TABLE 5. TESTING HRV INDICES IN “ADOLESCENT” (1) AND “EARLY YOUTH” (2) GROUPS AT THE CONTROL STAGE OF THE PEDAGOGICAL EXPERIMENT AFTER TRAINING ON VIBRATION SIMULATORS.}

Main indicators of heart rate rhythm

\begin{tabular}{|l|l|l|}
\hline & Value & Norm \\
\hline HR, bits/sec & 71 & $55-80$ \\
\hline SDNN, ms & 55.8 & $30-100$ \\
\hline CV (coefficient of variation), \% & 6.6 & $3-12$ \\
\hline SI (Stress Index), points & 114 & $50-150$ \\
\hline IC (Index of centralization) & $1.3 * *$ & $2-8$ \\
\hline NTI, points $(0,-2)$ & 2 & $1-3$ \\
\hline NArr, $\%$ & 0.0 & $0-4$ \\
\hline Strength HF, \% & $43.5^{* * *}$ & $10-30$ \\
\hline Strength LF, $\%$ & $50.2^{* * *}$ & $15-45$ \\
\hline Strength VLF, \% & $6.3^{* *}$ & $20-60$ \\
\hline TR, ms2 & $1745^{* *}$ & $800-1500$ \\
\hline
\end{tabular}

Atypical index (AI)

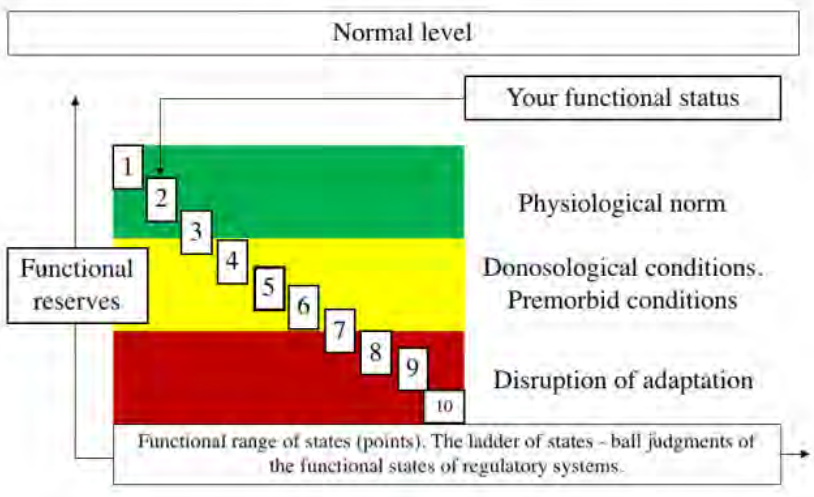

Assessment of the state of regulatory systems

\begin{tabular}{|c|c|}
\hline \multicolumn{2}{|l|}{ Level of functioning } \\
\hline Moderate bradycardia & -1 \\
\hline \multicolumn{2}{|l|}{ Stability of regulation } \\
\hline Normal rhythm stability & 0 \\
\hline \multicolumn{2}{|l|}{ Vegetative homeostasis } \\
\hline Normal vegetative balance & 0 \\
\hline \multicolumn{2}{|l|}{ Activity of the sympathetic vascular center } \\
\hline Normal activity of the vascular center & 0 \\
\hline \multicolumn{2}{|l|}{ Degree of centralization } \\
\hline Moderate decrease in activity of central levels of regulation & -1 \\
\hline
\end{tabular}

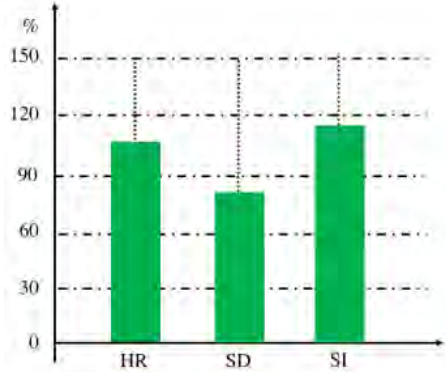

Fig. 2. Assessing the state of regulatory systems in the MG at the control phase of the pedagogical experiment.

Being combined and investigated, the MG 1 and 2 have the type I of cardiac rhythm regulation [7, 13, 
17], in which there is a moderate predominance of the influence of sympathetic and central regulation on CVS functions and a decrease in autonomic regulation of autonomic regulation (assessment of the functional state of regulatory systems by N. I. Shlyk) [17]. There is normalization at the control stage in the MG 1 and 2, elevated relative to the norm of the initial heart rate and stress index Si.

Stabilization of heart rate and stress index Si in the MG occurred due to the use of vibration platform in the training process, which helped to relieve muscle tension and improve the functioning of motor sensory systems. The works by E. M. Spivak [13] and N. I. Shlyk N. I. [17] note the participation and certain contribution of the genetic component to the determination of the type of vegetative regulation (VR) in children.

In our study, the dominance in the stasis phase of the 1st type of VR in the both MG and CG is due to a violation of the balance in the interaction between the regulatory and functional systems of the organism of young athletes. As a result of overtraining, they had reduced adaptive capabilities of the body, resulting in the depletion of the reserve capabilities of young athletes during training.

The classes with using vibroplatforms allowed to restore the balance of regulatory systems, the functional reserves of CVS, MS, to increase the stability and tolerance to the sports loads of the body of young handballers. As a consequence, we observe the transition to the 2 nd functional class at the control stage of the pedagogical experiment in the MG and CG, and Si parameters return to the norm (Table 5).

The results of the HRV study in the CG are shown in Table 6 and Figure 3.

TABLE 6. THE HRV INDICES BEING TESTED IN CG 1 AND 2 AT THE CONTROL STAGE.

Main indicators of heart rate rhythm

\begin{tabular}{|l|l|l|}
\hline & Value & Norm \\
\hline HR, bits/sec & $89^{* *}$ & $55-80$ \\
\hline SDNN, ms & 30.6 & $30-100$ \\
\hline CV (coefficient of variation), \% & 4.6 & $3-12$ \\
\hline SI (Stress Index), points & $316^{* *}$ & $50-150$ \\
\hline IC (Index of centralization) & 1.0 & $2-8$ \\
\hline NTI, points (2, 0) & 2 & $1-3$ \\
\hline NArr, \% & 0.0 & $0-4$ \\
\hline Strength HF, \% & $50.6^{* *}$ & $10-30$ \\
\hline Strength LF, \% & 29.4 & $15-45$ \\
\hline Strength VLF, \% & $19.9^{*}$ & $20-60$ \\
\hline TR, ms2 & 805 & $800-1500$ \\
\hline
\end{tabular}

Atypical index (AI)

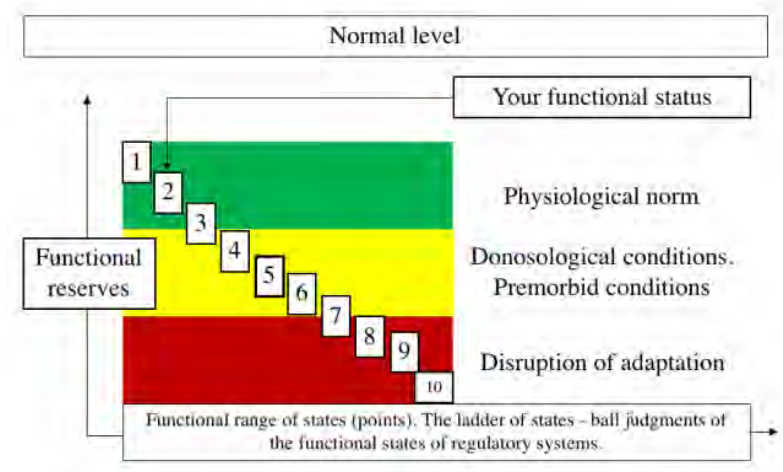

Assessment of the state of regulatory systems

\begin{tabular}{|c|c|}
\hline \multicolumn{2}{|l|}{ Level of functioning } \\
\hline Normocardia & 0 \\
\hline \multicolumn{2}{|l|}{ Stability of regulation } \\
\hline Expressed increase of the rhythm stability & 2 \\
\hline \multicolumn{2}{|l|}{ Vegetative homeostasis } \\
\hline Moderate predominance of the sympathetic nervous system & 2 \\
\hline \multicolumn{2}{|l|}{ Activity of the sympathetic vascular center } \\
\hline Moderately reduced activity of the vascular center & -1 \\
\hline \multicolumn{2}{|l|}{ Degree of centralization } \\
\hline Moderate reduce in the activity of central levels of regulation & -1 \\
\hline
\end{tabular}

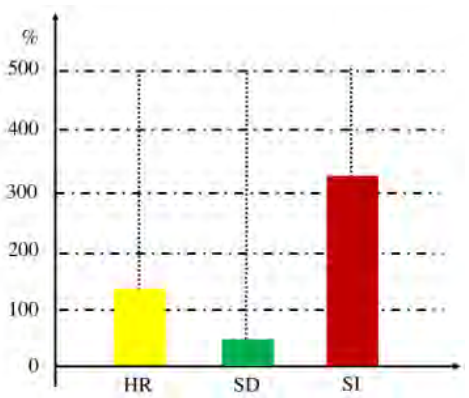

Fig. 3. Estimating the state of regulatory systems in the $\mathrm{CG}$ at the control stage.

In the CG, at the control stage of the study, there is also a second functional class corresponding to the physiological norm, but the heart rate and Si parameters did not approach the normal value. They remained overestimated, corresponding to the 1 st type of regulation of the heart rate (Table 6). In the CG, sportswomen were engaged in stretching during additional training in the post-training period without the use of a vibration platform. The results obtained in our study indicate that these effects are inadequate for restoring the reserve capacity of the organism and for the functional balance of the regulatory systems. Indicators of flexibility and coordination abilities in the $\mathrm{CG}$ are reduced in comparison with the $\mathrm{MG}$. 
Thus, the use of vibroplatforms in the post-training period contributes to the development of physical qualities (flexibility) and coordination abilities. Our studies have shown that this is facilitated by an increase in the functional reserves of athletes in the MG (Table 5, Figure 2), in contrast to the CG (Table 6, Figure 3), the improvement in muscle tone in the MG is of the I degree, in comparison with GS, where the II degree of muscle tone is observed. Therefore, the influence of the vibratory platform on young handballers can be attributed to multiplicative ones, in which the restoration of the reserve capabilities of the organism potentiates the developing and training effects of this method of post-training

\section{Conclusion}

The peculiarity of the physiological effect of the vibroplatform on the MS and the muscular system of the athletes consists in three-dimensional oscillations in the frontal, sagittal, and vertical planes. In this case, the muscles around the joints involved in the vibrational motion are involuntarily reduced to 30 times per $1 \mathrm{sec}$, with a frequency of $30 \mathrm{~Hz}$. In this case, the muscles are stretched better, more muscle fibers and muscle groups are actively involved, not participating in providing motor activities during training sessions. Activation of the contractile function of the muscles leads to an increase in their oxygen demand, which helps optimize the functions of the cardiorespiratory system to improve the blood supply to the muscles. The ordering and normalization of the CVS work and its regulatory mechanisms lead to the restoration of the reserve capabilities of the organism.

This contributes to increasing flexibility, coordination abilities, mobility in the joints, and proprioceptive sensory stability of athletes. Based on the conducted studies, it can be concluded that such a technology is effective for activating the processes of recovery and development of the organism of young athletes. Therefore, you can reasonably recommend the use of vibroplatforms in the training system of young female handball players.

\section{References}

[1] Antonov, I. P., Lupyan, Ya. A. (1986). Reference book on the diagnosis and prognosis of nervous diseases in tables and lists. Minsk, Belarus.

[2] Gazvan, G. M. (2011). Effective training tools that form the motivation to practice young handball players in the initial stages of preparation. Physical Culture and Health, 5, pp. 76-77.

[3] Grabovskaya, E. Yu., Belogub, N. V., Arkhangelskaya, E. V. (2018). The level of physical fitness and the development of motor skills in children of secondary school age in urban and rural schools. Scientific Notes of the Crimean Federal University named after V. I. Vernadsky. Biology. Chemistry, 4(70), 2, pp. 19-31.

[4] Eliseev, E. V., Evseev, A. V. (2017). The psychophysical potential of the physical development of handball players of 13-14 years old at the stage of early sports specialization. Orleu Bulletin, 1(15), pp. 130-135.

[5] Eliseev, E. V., Evseev, A. V. (2016). The development of the locomotor components of physical health of 11-13 years old handball players using vestibular training. Modern Problems of Science and Education, 6, pp. 471-483.

[6] Karpov, V. Yu., Maryina, N. V., Skorosov, K. K. (2015). Theoretical and methodological aspects of the related development of physical qualities and the formation of the technique of motor actions in girls of middle school age. Scientific Notes of the University named after P. F. Lesgaft, 6(124), pp. 91-96.

[7] Mavliev, F. A., Nazarenko, A. S., Sosnov, N. V. (2012). Typological features of variability of blood circulation parameters. Scientific Notes of the University named after P. F. Lesgaft, 9(91), pp. 97-101.

[8] Popovich A. P. (2013). Methods of education of the physical qualities of high school students in training groups on handball of children's and youth sports schools. Scientific notes of Ternopil National Pedagogical University named after Volodymyr Hnatyuk. Series: Pedagogy, 2, pp. 92-99. 
[9] Pyatin, V. F., Shirolapov, I. V. (2009). Physical activity by acceleration: the expansion of rehabilitation opportunities for regenerative medicine. Bulletin of Restorative Medicine, 1, pp. 25-29.

[10] Semenov, Yu. N. ()2014. Complex for processing cardiointervals and heart rate variability analysis "Varicard 2.51": Operation Guide. Ryazan: Ramena.

[11] Sitdikov, F. G., Sheikhelislamova M. V. (2008). Hormonal status and vegetative tone in children 7-15 years old. Kazan: TSHPU.

[12] Sosunovsky, V. S. (2014). Psychophysiological basis of gender differences in the development of physical qualities in children of middle school age. Bulletin of Science of Siberia,4(14), pp. 255-257.

[13] Spivak, E. M., Nezhkina, N. N. (2014). Features of the adaptation of the cardiovascular system to the load in young athletes with different types of vegetative regulation. Sports Medicine: Science and Practice, 1, pp. 32-36.

[14] Fomin, N. A., Vavilov, Yu. N. (1991). Physiological basis of motor activity. Moscow: Physical Culture and Sports.

[15] Tsyganok, A. V. (2007). The study of the integral indicators of the cardiovascular system of schoolgirls 10-16 years of age when systematically practicing handball. Pedagogy, Psychology and Biomedical Problems of Physical Education and Sport, 6, pp. 295-298.

[16] Tsyganok, A. V., Malikov, N. V. (2006). The influence of systematic handball on the nature of adaptive rearrangements of the cardiovascular system of the body in girls aged 10-16 years old. Physical Education of Students of Creative Specialties, 2, pp. 117-124.

[17] Shlyk, N. I. (2009). Heart rate and type of regulation in children, adolescents, and athletes. Izhevsk: Udmurt State University. 